\title{
Forecasting Mobile Internet Diffusion Trend Based on Optimized Bass Model
}

\author{
Zhaojie Zhu, Zhenhong Jia and Xizhong Qin \\ Xinjiang University \\ Chuanling Cao, China Mobile group Xinjiang Co., Ltd. \\ Chun Chang, China Mobile group Xinjiang Co., Ltd. \\ jsjzzj@163.com,jzhh@xju.edu.cn
}

\begin{abstract}
In order to make accurate forecast of mobile Internet diffusion trend. This paper proposes a method which is based on Bass innovation diffusion modet. To solve the problem that the parameters of the model are difficult to estimate, a Modified patricle swarm optimization algorithm (PSO) whose inertia weight changes dy namically is introduced to search the most precise parameters. The application of the index of population density helps determine the convergence status of population. When the algorithm is trapped into local optimum, the combination of Cauchy mutation and Gauss@m mutation is applied on the global best particle. The results of the experiment show that optimized Bassmodel is suitable for predicting mobile Internet diffusion trend and the proposed algorithm can effectively improve the precision of the optimal solution as compared to existed estimration methods.
\end{abstract}

Keywords: mobile Internet, particle swarm optimization, Bass model, hybrid mutation, forecasting

\section{Introduction}

The mobile Intemet has experienced rapid development in the recent years. There is an explosive growth trend of the revenues and the number of the subscribers. Predicting mobile Internet diffusion trend is mainly to predict the increasing trend of the number of subscribers. Given the rapid diffusion trend of mobile Internet industry, it is of great importance to research the increase of subscriber number in the past years and to forecast future growth potential. It can help enterprises which are related to mobile Internet industry to reduce the blindness as well as risk so as to make the correct decision. In addition, it can also provide advisory opinions to the telecommunication operators' meticulous, gridding and integrated network panning as well as business planning in order to further promote the efficiency of network resources allocation. The development process of mobile Internet is the application of an mnovation product essentially. Hence, the theory and model of innovation diffusion can be employed to research mobile Internet diffusion trend. It is reported that the Bass diffusion model was used to research the diffusion trend of various mobile Internet services [1]. The non-linear least squares was proposed in the literature for estimating the parameters in Bass model. Although this procedure is easy to implement, it has shortcoming with respect to providing high accuracy of forecast of diffusion trend.

The inertia weight in standard PSO decreases linearly which makes PSO incapable of successfully locating the global optimum in high dimensional objective functions. Arumugam and Rao proposed adaptive inertia weight techniques [2]. But the particle would probably oscillate around the optimum without or with low improvement. A new adaptive inertia weight strategy based on the success rate of the particles is proposed. The model proves the 
superiority over other inertia weight adjusting models [3]. However, it remains suffering from trapping into local optimum. Mutation operation can solve this problem effectively $[4,5]$. Based on above, we propose a method which is based on optimized Bass model to predict mobile Internet diffusion trend. A novel particle swarm optimization algorithm (HAPSO) with hybrid mutation and adaptively changed inertia weight is utilized to estimate the parameters. Experimental results illustrate the accuracy and efficiency of HAPSO as compared to existing PSO algorithms.

\section{Theoretical Framework}

\subsection{Bass Model}

Bass model depicts the increase trend in the number of adopters of a new product that incorporates external and internal influence. Thus potential adopters are divided into two different groups. One group is called innovators while the other imitators. The sum of innovators and imitators is the total number of adopters. Bass model supposes that there is no repeat adopters, which means that an adopter only buy one product [6-8]. In reality, however, an adopter of mobile Internet may have more than one mobile terminal that gets access to Internet. In the process of collecting statistical data the number of the subscribers is not defined as the number of Internet users, but the mobile terminals that have Internet access. The adoption of mobile Internet meets the assumption of Bass model by which the diffusion trend can be accurately predicted.

Parameters in Bass model have a greatimpact on the prediction accuracy. It is difficult to determine the appropriate values of parameters in/ad ance when applying the model to solve different practical problems. The values computed by different methods of parameter estimation varied greatly. It is critical to select the appropriate method to get the optimal parameters fitting the model best. Rajkunarar firstly proposed the application of genetic algorithm [9]. Compared with the traditional parameter estimation method, it gets a much better forecasting result. Nevertheless, this approach has the limitation that it is inefficient to search for the optinal solution, and each of iteration is executed at the cost of much time. Aiming at further imploving the aecuracy and speed up the convergent process, the novel HAPSO algorithm is applied to est mate the parameters.

\subsection{Modified PSO}

2.2.1. Dynamic Inertia Weight: PSO algorithm is simple in concept, easy to implement. It has spawned a wide range of applications since its introduction. Inertia weight $\mathrm{w}$ in standard PSO algorithm-decreases linearly according to the following equation:

$$
w=w_{\max }-\left(w_{\max }-w_{\min }\right)\left(\frac{t}{t_{\max }}\right)
$$

where $w_{\max }$ is the maximum of $w$ while $w_{\min }$ is the minimum, $t$ is the current iteration, $t_{\max }$ is the number of the total iterations. In this paper, $w_{\max }=0.9, w_{\min }=0.3, t_{\max }=100$.

Inertia weight $w$ has the capability of counterpoising global and local search abilities of the PSO. The larger value of $w$ can enable the PSO algorithm to obtain better global search ability. On the contrary, the smaller value of $w$ can ensure better local search ability. In the process of repeated iteration, $w$ is becoming smaller and smaller, thus PSO has better global search ability at the early phase of execution and better local search ability in the later phase. The widely application of inertia weight significantly improve convergent precision as well as efficiency. If the algorithm does not manage to find optimal solutions in the early phase, when it comes to the later phase, it is much likely to fall into local optimization for the reason that local search ability strengthens while global search ability as well as the diversity of whole 
swarm weaken. Currently, how should the inertia weight change remains lacking of exact theoretical guidance. A number of researchers have put forward many approaches to solve the problem [10-13]. According to their experimental results, the strategy that inertia weight changes dynamically can make PSO obtain better balance between global and local search abilities. We propose that the function of inertia weight can be defined as follows:

$$
w=\left(w_{\text {max }}-w_{\text {min }}\right) e^{-\frac{1}{n} \sum_{i=1}^{n}\left|\frac{f_{i-} f_{\text {avg }}}{f_{i}-f_{\text {best }}}\right|}+w_{\min }
$$

where $n$ is the number of particles, $f_{i}$ denotes the fitness value of $i$ th particle, $f_{\text {avg }}$ denotes the mean of all the fitness values, $f_{\text {best }}$ is the fitness value of the global best particle.

2.2.2. Hybrid Mutation: When the algorithm falls into local optima, Wang et al. and $\mathrm{Wu}$ utilized the mutation operator to increase the diversity of population. In the existed procedures, the probability that determines whether the mutation operator is conducted is randomly assigned to particles. This approach may lead to the particles which have been trapped into local optima cannot conduct mutation in time, thus resulting in the decrease of convergent speed. We introduced the index of population density to determine the timing of mutation, it is defined as follows:

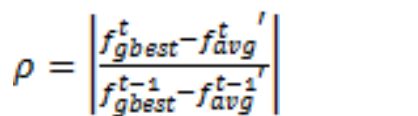

where $f_{\text {gbest }}^{\prime}$ denotes the fitness value of the global best particle in $t$ generation. $f_{\text {avg }}^{\prime}$ ' is mean of the fitness values of the particles whose fitness values are greater than the average values of the population. The Cauchy mutation and Gaussian nutation have their own advantages. We propose the combination of them is:

$$
x_{j}^{\prime}=x_{j}+\left(b_{j}-a_{j}\right)((1-\lambda) \eta+\lambda \vartheta), p \leq \varepsilon \text { and } f_{g b s s t}^{k}-f_{d} \geq \delta
$$

where $\lambda$ is a parameter in [0, 1], $\eta$ is a random number generated by the standard Cauchy distributed function, $\theta$ is $\alpha$ standard Gaussian distribution number. $\varepsilon$ and $\delta$ are positive constants close to 0 , when the value of $\rho$ is smaller than threshold $\varepsilon$, it means that the algorithm is in the status of convergence. Then if the value of $f_{g b e s t}^{t}-f_{d}$ is greater than threshold $\delta, f$ is expected optimal solution. It is clear that the algorithm is falling into local optima, and the mutation can be conducted. Otherwise the standard PSO is executed. $b_{j}-a_{j}$ can be considered as the size of the search space. In the early evolution, a large range of disturbances can be applied on $x_{j}$ to enhance global search ability. With the reduction of $b_{j}-a_{j}$, the local search ability is better. The limitation of Bass model is that the three parameters in model are hard to determine. Define three- dimensional particles as the possible optimal solution of the parameters. Then PSO is employed to search for the optimums that are the optimal parameters for Bass model.

Steps of searching for the optimum can be described as follows:

1) Initialize PSO algorithm within the search space.

2) Calculate each particle's fitness value that is calculated by objective function

3) Update the personal best position for each particle and the global best position for the population.

4) Update the velocity and position of each particle.

5) Judge if the algorithm falls into local optima according to formula (3), go to the next step. Otherwise go to step 7.

6) Hybrid mutation is conducted on global best particle, go to step 2

7) Judge whether the termination criterion is satisfied. If the criterion is met, then stop.

Otherwise, go to step 2.

8) Introduce the optimal parameters into Bass model and make the forecast. 


\section{Experiment and Result Analysis}

The experimental data for mobile Internet subscribers of every month from January 2010 to July 2013 is obtained from branch of China Mobile in a certain city. The statistical data is divided into a training set containing the first 36 data and a testing set containing the later 7 data. The increasing trend of mobile Internet subscribers who belong to the China Mobile company will be predicted.

Initialization of PSO algorithm: the scale of swarm is 40. The solution space is three-dimensional. The maximum of iteration is 100. $c_{1}=c_{2}=2, \omega_{\max }=0.9, \omega_{\min }=0.3, \varepsilon=0.05$, $f_{d}=0, \delta=10^{-5}, \lambda=0.8$. The parameters setting of Bass model are following: innovation coefficient $p$ and imitation coefficient $q$ are certain values between 0 and 1 . According to the Statistical Yearbook of this city issued in 2013, the population is 0.5 million with the growth trend continues. The penetration rate of mobile phone has reached $100 \%$. The China Mobile occupies the market share of about $70 \%$. Thus the total latent subscribers can be considered to belong to the range [200000, 50000].

In order to evaluate the performance of the HAPSO algorithm, the forecasting results generated from PSO, AIWPSO [3] are compared. The results are illustrated in Figure 1:

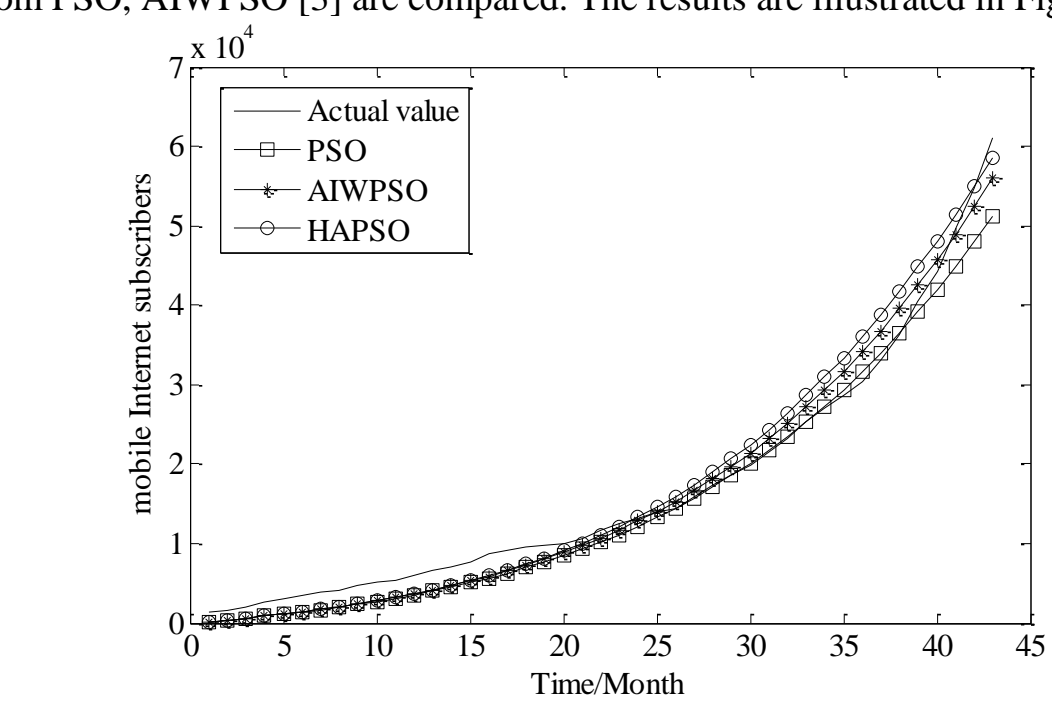

Figure1, Forecasting Results of Different Algorithms

The evaluation indices of error use Mean Absolute Percent Error (MAPE) and Root Mean Square Error (RMSE). The value of parameters and the forecasting error calculated by different algorithms are shown in Table 1 :

\section{Table 1. Comparisons of Parameters and Forecasting Error}

\begin{tabular}{cccccc}
\hline \multirow{2}{*}{ Algorithm } & \multicolumn{3}{c}{ Parameter } & \multirow{2}{*}{ MAPE } & \multirow{2}{*}{ RMSE } \\
& $p$ & $q$ & $m$ & & \\
\hline PSO & 0.00082 & 0.0845 & 228997 & $8.49 \%$ & 3869 \\
AIWPSO & 0.00065 & 0.0793 & 288792 & $6.41 \%$ & 3062 \\
HAPSO & 0.00053 & 0.0776 & 348944 & $5.67 \%$ & 2158 \\
\hline
\end{tabular}

Analysis of Figure 1and Table 1, some important conclusions can be drawn:

The forecast of the number of mobile Internet subscribers is much closed with the actual value, and the goodness of fit of Bass model reaches a high degree, the least value of MAPE and RMSE are 5.67\% and 2158 respectively. Hence the application of Bass model on forecasting diffusion trend of mobile Internet is reasonable. 
The forecasting error calculated by PSO is the biggest compared to the other approaches. It proves that the strategy that inertia weight changes dynamically can make PSO obtain better performance in comparison to linearly change strategy. Compare PSO, AIWPSO with HAPSO, the proposed algorithm is found to achieve the best performance in terms of improving the precision of forecast, which means that mutation operation solves this problem of local optimum effectively. It is a suitable approach to estimate the parameters in Bass model.

\section{Conclusions}

The application of Bass model to predict mobile Internet diffusion trend is demonstrated in this paper. In the proposed method the parameters are estimated by a modified PSO algorithm in which inertia weight is dynamically adjusted and hybrid mutation is used to escape from local optimum. The proposed method is tested on statistical data and compared with other methods. The results show that Bass model can accurately predict the diffusion trend. The novel algorithm obtains a better balance between global and local seareh abilities for better performance on forecasting. The current number of subscribers are far from reaching the maximum of potential adopters. Mobile Internet will develop rapidiy in the next few years.

\section{Acknowledgments}

We gratefully thank the financial support by China Mobile group Xinjiang Co., Ltd (XJM2013-2788).

\section{References}

[1] Y. Li, "Research on the diffusion model of mobileInternet service and its application", Beijing University of Posts and Telecommunications, (2011).

[2] M. S. Arumugam and M V.C. Rao, "On the improved performances of the particle swarm optimization algorithms with adaptive parameters", cross-over operators and root mean square (RMS) variants for computing optimal contrel of a class of hybrid systems, Applied Soft Computing, vol. 8, no. 1, (2008).

[3] A. Nickabadi, M. Mehd Ebadzadeh and R. Safabakhsh, "A novel particle swarm optimization algorithm with adaptive inertia weight", Applied Soff Computing, vol. 11, (2011).

[4] H. Wang, Z. J. Wu, S. Rahnamayan, "Enhancing particle swarm optimization using generalized opposition-based learning", Information Sciences, vol. 181, (2011).

[5] Q. Wu, "Cauchy mutation for decision-making variable of Gaussian particle swarm optimization applied to parameters selection of SVM", Expert Systems with Applications, vol. 38, (2011).

[6] L. Diao, B. Zhang and L. Jia, "Diffusion of information technology: an extension of the Bass model", Proceedings of International Symposium on Information Engineering and Electronic Commerce, (2009) May 16-17, Washíngton, DC, USA.

[7] A. Wang and H. Hao, "E-commerce research with Bass product diffusion model. Proceedings of International Conference) on E-Business and E-Government", (2010) May 7-9, Guangzhou, China.

[8] J Yang and W. Wei, "Research on the differences of three s-shaped curves based on Genetic algorithm", Proceedings of the 2nd International Conference on Information Engineering and Computer Science, (2010) Deeember 25-26, Wuhan, China.

[9] R. Venkatesan and V. Kumar, "A genetic algorithms approach to growth phase forecasting of wireless subscribers. International Journal of Forecasting, vol. 18, (2002).

[10] Z. Yan, C. Deng, J. Zhou and D. Chi, “A novel two-subpopulation particle swarm optimization”, Proceedings of the 10th World Congress on Intelligent Control and Automation, (2012) July 6-8, Beijing, China.

[11] M. Chen, T. Wang and J. Feng, "A hybrid particle swarm optimization improved by mutative scale chaos algorithm", Proceedings of the Fourth International Conference on Computational and Information Sciences, (2012) August 17, Chongqing, China.

[12] M. Hu, T. Wu and J. D. Weir, "An intelligent augmentation of particle swarm optimization with multiple adaptive methods", Journal of Information Sciences, vol. 5, (2012).

[13] M. Gang, W. Zhou and X. Chang, "A novel particle swarm optimization algorithm based on particle migration", Journal of Applied Mathematics and Computation, vol. 218, no. 11, (2012). 


\section{Authors}

Zhaojie Zhu, He received his BE in Computer Science from Xinjiang Agriculture University in 2011. He is now a graduate student in Xinjiang University. His research interests include machine learning and mobile Internet.

Zhenhong Jia, He received his BE from Beijing Normal University in1986. He received his ME and PhD from Shanghai Jiaotong University in 1989 and 1993. Currently, he is with the Xinjiang University where he is a Professor and a PhD tutor of information science. His research interests include optical communications, wireless communications. He has published over 100 papers. He was select for new century excellent talents plan in 2006. He is a member of Chinese Optical Society of Holography and Optical Information Processing Committee.

Xizhong Qin, He received his ME from Southeast Uniyersity in 1990 . Currently, he is with the Xinjiang University where he is an associate Professor and a Master tutor of information science. His research interests include signal processing, wireless communications.

Chuanling Cao, She is an employee of China Mobile group Xinjiang Co., Ltd. Her major is service planning.

Chun Chang, He is an employeefof'China Mobile group Xinjiang Co., Ltd. His specialty is network planning. 